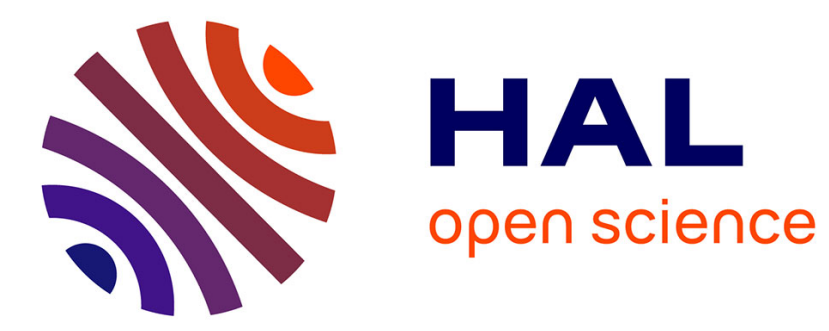

\title{
Diffusion Raman stimulée dans une fibre en silice dopée au phosphore
}

\author{
A. Saïssy, A. Azema, J. Botineau, F. Gires
}

\section{To cite this version:}

A. Saïssy, A. Azema, J. Botineau, F. Gires. Diffusion Raman stimulée dans une fibre en silice dopée au phosphore. Journal de Physique Lettres, 1979, 40 (2), pp.23-25. 10.1051/jphyslet:0197900400202300 . jpa-00231560

\section{HAL Id: jpa-00231560 https://hal.science/jpa-00231560}

Submitted on 1 Jan 1979

HAL is a multi-disciplinary open access archive for the deposit and dissemination of scientific research documents, whether they are published or not. The documents may come from teaching and research institutions in France or abroad, or from public or private research centers.
L'archive ouverte pluridisciplinaire HAL, est destinée au dépôt et à la diffusion de documents scientifiques de niveau recherche, publiés ou non, émanant des établissements d'enseignement et de recherche français ou étrangers, des laboratoires publics ou privés. 


\title{
Diffusion Raman stimulée dans une fibre en silice dopée au phosphore
}

\author{
A. Saïssy, A. Azema, J. Botineau et F. Gires \\ Laboratoire d'Electrooptique (*), Parc Valrose, 06034 Nice Cedex, France. \\ (Reçu le 26 octobre 1978, révisé le 20 novembre 1978, accepté le 22 novembre 1978)
}

\begin{abstract}
Résumé. - Le spectre de diffusion Raman stimulée d'une fibre optique à cœur de silice dopée au phosphore présente une raie à $1320 \mathrm{~cm}^{-1}$ que nous attribuons à la présence du phosphore. L'étude de la diffusion spontanée et la mesure directe du gain à l'aide d'un amplificateur à fibre confirment l'origine de la raie.
\end{abstract}

Abstract. - The stimulated Raman scattering spectrum of an optical fiber with a phosphate doped silica core has a line at $1320 \mathrm{~cm}^{-1}$ which we attribute to the phosphate. The study of the spontaneous scattering and the direct measurement of the gain with a fiber amplifier confirm the origin of the line.

1. Introduction. - L'utilisation de fibres optiques pour l'étude d'effets non linéaires est très avantageuse car elle permet de conserver une intensité lumineuse importante sur une grande longueur [1,2]. Dans le cas de la diffusion Raman stimulée cette longueur d'interaction importante permet de compenser le faible rendement Raman de la silice.

D'autre part la fabrication d'une fibre optique à partir de la silice nécessite un dopage de cette dernière. Le plus souvent le type de dopant employé est $\mathrm{B}_{2} \mathrm{O}_{3}$ qui abaisse l'indice pour la gaine et $\mathrm{GeO}_{2}$ ou $\mathrm{P}_{2} \mathrm{O}_{5}$ qui augmentent l'indice pour le cœur.

Le rendement d'un générateur Raman à fibre sera différent suivant que la fibre possède un cour dopé au germanium ou au phosphore. Dans la présente note nous étudions le rôle du phosphore dans le fonctionnement d'un tel dispositif.

2. Diffusion Raman stimulée. - Dans un premier temps nous avons réalisé un générateur Raman à l'aide d'une fibre à saut d'indice. Le cour dopé à $5 \%$ de $\mathrm{P}_{2} \mathrm{O}_{5}$ a un diamètre de $50 \mu \mathrm{m}$, la longueur de la fibre est soit $12 \mathrm{~m}$ soit $50 \mathrm{~m}$. La longueur d'onde du faisceau excitateur est $5320 \AA$; il est fourni par un laser YAG pulsé (impulsion de durée $10 \mathrm{~ns}$ ) fonctionnant à $1,06 \mu \mathrm{m}$ suivi d'un cristal de KDP doubleur de fréquence. La puissance disponible à $5320 \AA$ est de quelques dizaines de kilowatts. Le schéma de principe du montage est représenté sur la figure 1 .

(*) Associé au C.N.R.S. no 190.

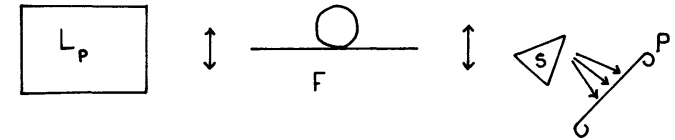

Fig. 1. - Générateur Raman à fibre : $L_{p}=$ laser YAG et KDP, $F=$ fibre, $S=$ spectrographe, $p=$ plaque photographique.

[Fiber Raman generator : $\mathrm{L}_{\mathrm{p}}=\mathrm{YAG}$ laser and $\mathrm{KDP}, \mathrm{F}=$ fiber, $\mathrm{S}=$ spectrograph, $\mathrm{p}=$ photographic plate.]

La figure 2 représente quelques exemples de spectres obtenus avec une fibre de $12 \mathrm{~m}$. Sur ces spectres on distingue du côté Stokes de la radiation excitatrice

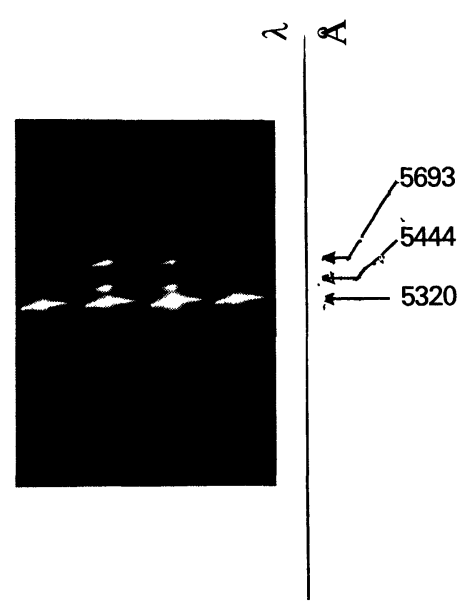

Fig. 2. - Spectre de diffusion Raman stimulée.

[Stimulated Raman scattering spectra.] 
deux raies : une première $v_{1} \sim 440 \mathrm{~cm}^{-1}$ suivie d'une seconde $v_{2} \sim 1320 \mathrm{~cm}^{-1}$. La raie à $440 \mathrm{~cm}^{-1}$ correspond à une vibration caractéristique de la silice [3]. La présence de la raie à $1320 \mathrm{~cm}^{-1}$ ne peut s'interpréter comme un processus de cascade multistokes : le deuxième Stokes manque et la relation $v_{2}=3 v_{1}$ n'est pas vérifiée. La résolution des clichés photographiques ne permet pas une étude spectrale fine de nos spectres. Pour effectuer cette étude nous avons remplacé notre caméra par une mosaïque de photodiodes au silicium et utilisé une fibre de $50 \mathrm{~m}$ avec un cœur de $7 \mu \mathrm{m}$. La figure 3 reproduit un spectre particulier pour lequel le processus de cascade multistokes coexiste avec la diffusion à $1320 \mathrm{~cm}^{-1}$. On distingue

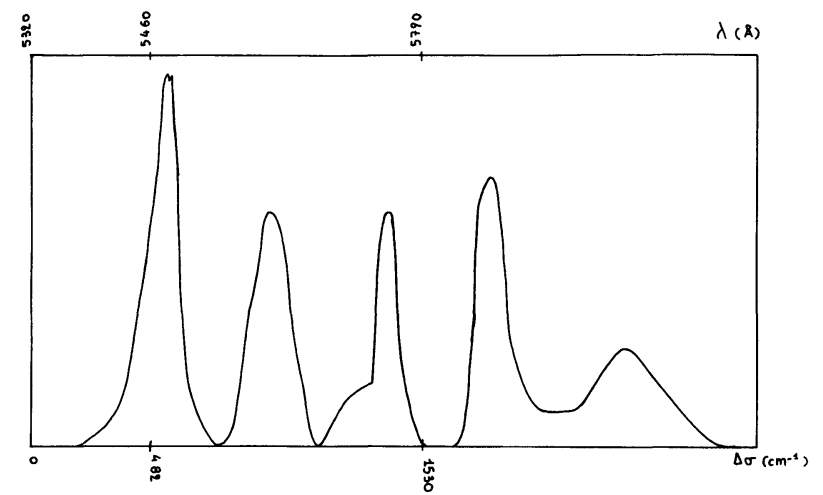

Fig. 3. - Analyse spectrale d'un spectre de diffusion Raman stimulée.

[Spectral scanning of a stimulated Raman spectra.]

une première raie Stokes à $(540 \pm 60) \mathrm{cm}^{-1}$ donnant naissance à une seconde et une troisième raie Stokes, sur le flanc descendant de cette troisième raie se superpose une raie plus intense et plus étroite à $(1400 \pm 60) \mathrm{cm}^{-1}$, c'est la deuxième raie du spectre de la figure 2. Il apparait donc que l'onde excitatrice bénéficie d'un gain non nul à $1320 \mathrm{~cm}^{-1}$. La présence d'un tel gain $G_{\text {s }}$ proportionnel à la section efficace de diffusion Raman spontanée $\sigma$ doit correspondre à l'apparition d'une bande dans le spectre de diffusion Raman spontanée conformément à la relation

$$
G_{\mathrm{s}}=\sigma \lambda_{\mathrm{s}}^{3} / C^{2} h \varepsilon(n+1)
$$

d'où $\lambda_{\mathrm{s}}=$ longueur d'onde Stokes, $h=$ constante de Planck, $\varepsilon=$ constante diélectrique, $n=$ facteur de population de Bose-Einstein.

3. Diffusion Raman spontanée. - Nous avons utilisé une fibre en silice à saut d'indice à cœur dopé au phosphore de longueur $50 \mathrm{~m}$ et de diamètre de cœur $50 \mu \mathrm{m}$. Un laser argon réglé sur $5145 \AA$ est couplé dans cette fibre, à la sortie le spectre de la lumière est étudié à l'aide d'un spectromètre Ramanor Jobin-Yvon suivi d'une chaîne de photocomptage. Les mesures de diffusion Raman en sortie de fibre permettent de bénéficier de toute la longueur de la fibre et de ne considérer que le matériau constituant son cœur, les mesures de diffusion à $90^{\circ}$ n'offrent pas ces avantages. La figure 4 représente les spectres de la silice pure et de la fibre, la bande à $440 \mathrm{~cm}^{-1}$ est présente dans les deux cas; par contre seule la fibre possédant du phosphore possède une bande à $1320 \mathrm{~cm}^{-1}$. Le spectre de $\mathrm{P}_{2} \mathrm{O}_{5}$ pur possède une bande à $1390 \mathrm{~cm}^{-1}$ identifiée comme étant une vibration d'élongation de la liaison $\mathrm{P}=\mathrm{O}$ [4]. La bande à $1320 \mathrm{~cm}^{-1}$ de notre spectre est probablement due à la vibration de la liaison $\mathrm{P}=\mathrm{O}$ lorsque le phosphore est noyé dans la matrice cristalline du $\mathrm{SiO}_{2}$. Nous confirmons ainsi l'existence d'un gain non nul à $1320 \mathrm{~cm}^{-1}$ dans la silice dopée au phosphore, son origine étant la vibration de la double liaison $\mathrm{P}=\mathrm{O}$. En utilisant cette vibration il est donc possible de construire un amplificateur Raman fonctionnant au voisinage de $1320 \mathrm{~cm}^{-1}$.

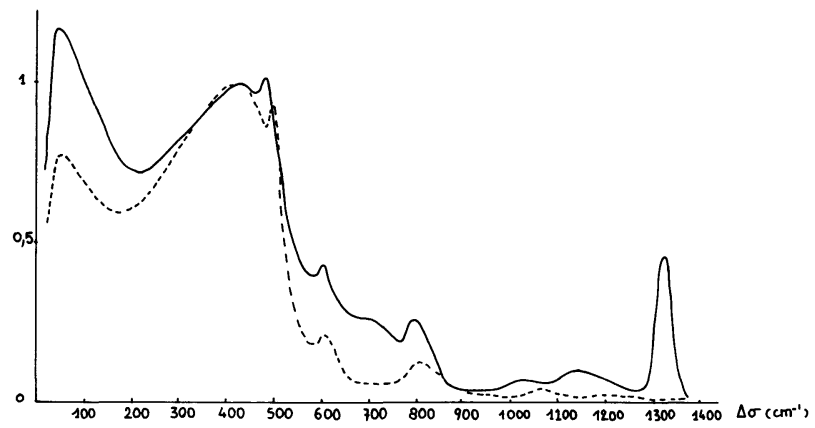

Fig. 4. - Spectres de diffusion Raman spontanée : — fibre de silice dopée au phosphore; -...- silice pure.

[Spontaneous Raman scattering spectra : — phosphate doped silica fiber ; ..... pure silica.]

4. Amplificateur Raman. - Une onde lumineuse intense $\mathbf{E}_{\mathbf{L}}\left(\omega_{\mathbf{L}}\right)$ de pulsation $\omega_{\mathbf{L}}$ se propageant dans un milieu Raman actif induit un gain à la pulsation $\omega_{\mathrm{S}}=\omega_{\mathrm{L}}-\omega_{\mathrm{V}}\left(\omega_{\mathrm{V}}=\right.$ pulsation vibrationnelle de la molécule de silice). Une onde $\mathbf{E}_{\mathbf{S}}\left(\omega_{\mathbf{S}}\right)$ de pulsation $\omega_{\mathbf{S}}$ se propageant avec $\mathbf{E}_{\mathbf{L}}\left(\omega_{\mathbf{L}}\right)$ permet de mesurer ce gain. Pour réaliser un tel amplificateur nous avons utilisé une fibre en silice dopée au phosphore fabriquée par la C.G.E. Ses diamètres externe et interne sont 80 et $7 \mu \mathrm{m}$ respectivement, sa longueur est $50 \mathrm{~m}$, les pertes sont de $24 \mathrm{~dB} \mathrm{~km}^{-1}$ à $6328 \AA$. Le schéma de principe est représenté sur la figure 5 . Le faisceau lumineux servant à pomper la fibre est fourni par un laser à colorant de largeur de raie $\Delta \omega_{\mathrm{e}}<15 \mathrm{~cm}^{-1}$ (la largeur de la bande à $1320 \mathrm{~cm}^{-1}$ est

$$
\left.\Delta \omega_{\mathrm{S}} \simeq 50 \mathrm{~cm}^{-1}>\Delta \omega_{\mathrm{e}}\right)
$$

délivrant des impulsions de durée $10 \mathrm{~ns}$ et de longueur d'onde variable. Le faisceau lumineux dont on mesure l'amplification est fourni par un laser $\mathrm{HeNe}$ réglé à $6328 \AA$. Les deux faisceaux sont couplés simulta- 


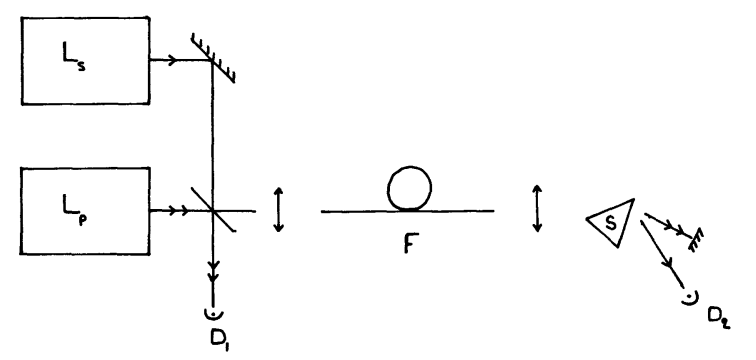

Fig. 5. - Amplificateur Raman à fibre : $\mathrm{L}_{\mathbf{s}}=$ laser $\mathrm{HeNe}$, $\mathrm{L}_{\mathrm{p}}=$ laser colorant, $\mathrm{F}=$ fibre, $\mathrm{S}=$ spectrographe, $\mathrm{D}_{1}, \mathrm{D}_{2}=$ détecteurs.

[Fiber Raman amplifier : $\mathrm{L}_{\mathrm{S}}=\mathrm{HeNe}$ laser, $\mathrm{L}_{\mathrm{p}}=$ dye laser, $\mathrm{F}=$ fiber, $\mathrm{S}=$ spectrograph, $\mathrm{D}_{1}, \mathrm{D}_{2}=$ detectors.]

nément dans la fibre, un premier détecteur $\mathrm{D}_{1}$ donne un signal $I_{\mathrm{p}}$ proportionnel à l'intensité de pompe à l'entrée de la fibre. Un spectrographe suivi d'un monochromateur placé à la sortie de la fibre permet de ne conserver que la radiation à $6328 \AA$, le détecteur $\mathrm{D}_{2}$ permet de mesurer son intensité $I_{\mathrm{s}}$. Les signaux $I_{\mathrm{p}}$ et $I_{\mathrm{S}}$ sont affichés sur un oscilloscope à mémoire, on calcule le gain $G$ à partir de la formule

$$
I_{\mathrm{S}}=I_{\mathrm{S}_{0}} \exp G I_{\mathrm{p}} l
$$

pour chaque intervalle de pulsation $\Delta \omega=\omega_{\mathrm{L}}-\omega_{\mathrm{S}}$. La figure 6 montre une telle courbe normalisée à $1320 \mathrm{~cm}^{-1}$, on constate un maximum vers $1320 \mathrm{~cm}^{-1}$ avec une dispersion appréciable.

En effet la fibre n'est pas exactement monomode, les ondes de pompe et de sonde peuvent changer de mode de propagation en cours de manipulation ce qui introduit des fluctuations sur le signal mesuré. Néanmoins aux imprécisions de mesure près on constate la présence d'une bande d'amplification centrée sur $1320 \mathrm{~cm}^{-1}$. Ce qui confirme les résultats précédents.

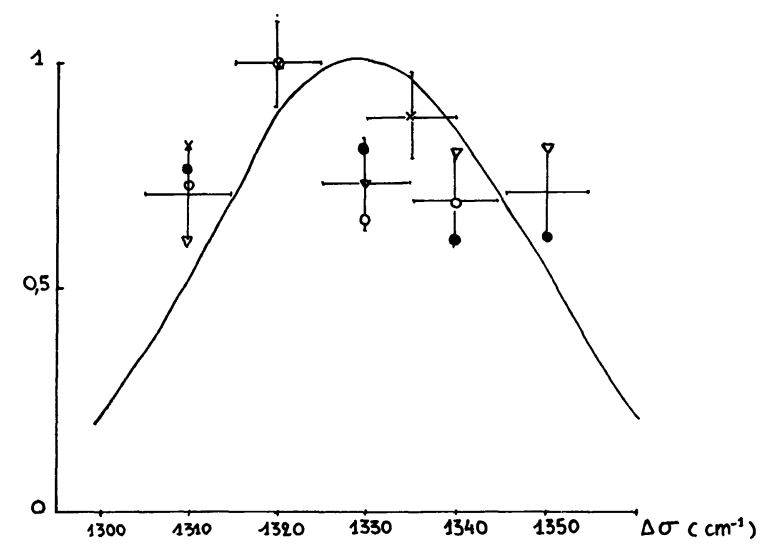

Fig. 6. - Courbe de gain : $\bigcirc, \bullet, \nabla$ points expérimentaux; spectre spontané.

[Gain curve : $\bigcirc, \bullet, \nabla$ experimental points; - spontaneous spectra.]

5. Conclusion. - Nous avons montré expérimentalement que la présence du phosphore même en faible quantité (quelques centièmes) dans le cour d'une fibre donne lieu à un gain Raman supplémentaire centré sur $1320 \mathrm{~cm}^{-1}$, gain inexistant dans la silice pure et dans la silice dopée au germanium. Deux applications nous paraissent envisageables :

- d'une part en étudiant la variation spatiale de l'émission spontanée à $1320 \mathrm{~cm}^{-1}$ on peut tracer des profils de dopage et déterminer des concentrations en phosphore (des mesures de ce type ont été effectuées sur des préformes, pour des fibres le problème paraît plus délicat);

- d'autre part la réalisation d'un amplificateur à fibre fonctionnant à $1320 \mathrm{~cm}^{-1}$ permet d'envisager la réalisation d'un oscillateur à fibre fonctionnant à $1320 \mathrm{~cm}^{-1}$ avec un niveau de pompe plus faible qu'un oscillateur à silice basé sur la création de trois raies Stokes successives.

\section{Bibliographie}

[1] Stolen, R. H., Ippen, E. P., Tynes, T. R., Appl. Phys. Lett. 20 (1972) 62.

[2] Botineau, J., Gires, F., Saïssy, A., Vanneste, C., Azema, A., Appl. Opt. 17 (1978) 1208.

[3] Stolen, R. H., Walrafen, G. E., J. Chem. Phys. 64 (1976) 2623.

[4] Galeener, F. L., Mikkelsen, J. C. Jr, Geils, R. H., Mosby, W. J., Appl. Phys. Lett. 32 (1978) 34. 